\title{
A study on Contact Algebra by Browerian Logic
}

\author{
Surendra Raj Pathak ${ }^{*}$, Raj Narayan Yadav ${ }^{2}$ \\ ${ }^{1}$ Department of Mathematics, Amrit Campus, Kathmandu \\ Tribhuvan University, Nepal \\ ${ }^{2}$ Department of Mathematics, MMAM Campus, Biratnagar \\ Tribhuvan University, Nepal \\ *Email : pathaksr2030@gmail.com
}

(Received: 20 April, 2020, Received in revised form: 2 August, 2020, Accepted:10 August, 2020, Available online)

\section{Highlights}

- Close relation between Boolean logic and an electric dicontact algebra has been studied.

- ON and OFF operators on a list $\mathrm{L}$ are defined and established some results involving them.

- Fuzzy model of contact algebra has been in introduced and the properties are evaluated.

- Contact algebra has been reviewed by Browerian Logic,

\begin{abstract}
There is a close relation between Boolean logic or two-valued logic and an electric di-contact algebra. Two-valued logic is concerned with propositions which are either true or false and which can be combined in various ways. Similarly, the switches of circuits are activated by contacts which, open or closed, can be combined in analogous ways. But there are positions which are not two-valued - a generalisation of truth values of a proposition leads to an n-valued logic. It is then natural to raise the query whether it is possible to generalise the notion of switching contact analogous to the generalisation of truth value of a proposition. If it is so, does there exist an isomorphism between propositional algebra in n-valued logic and a structure in switching circuits based on contact values? The solution of the problems leads to a new algebra. Here we have reviewed this contact algebra by Browerian logic.
\end{abstract}

Keywords: Boolean logic, n-valued logic, Fuzzy model, Browerian logic

\section{Introduction}

Boolean circuits are used to represent programmes on finite data. Its origin is found in Boole's mid-nineteenth century work on classical logic and the success of what we now call Boolean algebra. It led to the late nineteenth century attempts for the formalization of all mathematical reasoning and eventually to mathematical logic (Wallman, 1938). Lafont introduced reversible Boolean circuits and quantum Boolean circuits (Lafont, 2003 and Lafont 2004). He considered logic gates and generators for some algebraic structures with two compositions (Steiner, 1966). He was actually interested in the relations satisfied by these generators. For that purpose he introduced canonical forms and rewriting systems to get presentation of generators and relations for the classical case as well as quantum. These notions are essential in complexity theory but his ultimate conclusion was that a deep mathematical theory is needed to make progress in this field (Meckinsey et al, 1947).

${ }^{*}$ Corresponding author 


\section{Definitions}

In $n$-valued logic the truth value of a proposition is not always false and a contact different from 'on' will never break the circuit. Therefore, we define the following contact algebra.

Definition 2.1. A contact algebra $(C, \wedge, \vee)$ over an electric circuit is a distributive lattice of finite contacts with the operations of connection in series $(\wedge)$ and in parallel $(\vee)$.

A di-contact algebra is a chain in which $x \vee y=\max (x, y)$ and $x \wedge y=\min (x, y)$. But in $n$-contact algebra any two contacts $x$ and $y$ are not necessarily comparable. However, it is possible to introduce Browerian operation B or a dual Browerian operation $\mathrm{B}^{*}$ to tackle the problems (Birkhoff, 1984).

Definition 2.2. Let $x, y \in C$. Then we say that $x$ is Browerian with $y$ i.e. $x$ B $y$ iff $x \wedge z \leq y$ for some $z \in C$.

Dually $x \mathrm{~B}^{*} y$ iff $x \vee t \geq y$ for some $t \in C$.

Let $x, y \in C$ and lęt $z_{i}$ be the contacts such that $x \wedge z_{i} \leq y$ for each $i[i=1,2,3, \ldots, n]$. Then $x \bigwedge_{i=1}^{n}\left(\bigvee z_{i}\right) \leq y$. Since $\bigvee_{i=1}^{n} z_{i}$ exists and is unique, $\bigvee_{i=1} z_{i}$ is the Browerian contact with respect to $y$ and is denoted by $x \rightarrow y$. The dual Browerian contact of $x$ with respect to $y$ is the contact $\bigwedge_{i=1}^{n} t_{i}$ for which $x \vee\left(\wedge t_{i}\right) \leq y$ and is denoted by $x \leftarrow y(R . N$ Lal et al, 1998).

Definition 2.3. The complement $x^{*}$ of a contact $x$ in $C$ is defined as

$$
x^{*}=x \rightarrow 0
$$

Definition 2.4. The duel complement $\bar{x}$ of a contact $x$ in $C$ is defined as

$$
\bar{x}=x \leftarrow 1
$$

\section{Theorems}

Theorem 3.1. The Browerian relation $\mathrm{B}$ on $(C, \wedge, \vee)$ is prelinear ordering.

Proof. (i) $x \wedge l<x \Rightarrow x \mathrm{~B} x, \forall x \in C$

(ii) $x \mathrm{~B} y$ and $y \mathrm{~B} z$

$\Rightarrow x \wedge u<y$ and $y \wedge v<z$ for some $u, v$ in $C$

$\Rightarrow x \wedge y \wedge u \wedge v<y \wedge z$

$\Rightarrow x \wedge u \wedge v<z$

[cancellation holds]

$\Rightarrow x \wedge w<z$ $[w=u \wedge v]$

$\Rightarrow x \mathrm{~B} z$

A Browerian relation induces the notion of Browerian operation in an electric circuit.

Theorem 3.2. $(C, \wedge, \vee)$ is a Browerian logic.

Proof. It is because $(C, \wedge, \vee)$ is a finite distributive lattice in which

$$
\begin{aligned}
& (\bar{x}) \leq x \\
& (\bar{x})=(\overline{\bar{x}})=\ldots \\
& (\bar{x})=\left(\begin{array}{l}
\equiv \\
x
\end{array}\right)=\ldots
\end{aligned}
$$


Remark 3.1. $(C, \wedge, \vee)$ is a Boolean iff $\bar{x}^{*}=x$.

Theorem 3.3. (a) Every rational number and in particular non-zero and non-negative generates a contact algebra.

(b) A set of rational numbers and in particular that of integers closed with respect to the highest common factor and the lowest common multiple is a contact algebra (Bolyar, 1981).

(c) Lukasiewicz as well as Rosser truth values of a proposition determine a contact algebra .

Proof. (a) Let $r_{0}=(r), r_{1}, r_{2}, r_{3}, \ldots, r_{n}$ are $n$ factors of $r$ and $r_{n}$ the H.C.F. of all the factors. Then the set $L=\left\{r_{0}, r_{1}, r_{2}, r_{3}, \ldots, r_{n-1}\right.$, $r_{n}$ \} under the relation of divisibility is a contact algebra. Furthermore, if the factors of $r$ are taken in such a manner that $r_{i} \mid r_{i+1}$, $i=0,1,2,3, n-1$, then the contact algebra is a chain

(b) Obvious.

(c) The set of Lukasiewicz truth values under usual order is clearly a contact algebra in which a matrix for $\rightarrow$ is given by :

\begin{tabular}{|c|c|c|c|c|c|c|}
\hline$\rightarrow$ & 0 & $1 /(\mathrm{n}-1)$ & $2 /(\mathrm{n}-1)$ & $\ldots$ & $(\mathrm{n}-2) /(\mathrm{n}-1)$ & 1 \\
\hline 0 & 1 & 1 & 1 & $\ldots$ & 1 & 1 \\
\hline $1 /(\mathrm{n}-1)$ & 0 & $\mathrm{I}$ & 1 & $\ldots$ & 1 & 1 \\
\hline $2 /(\mathrm{n}-1)$ & 0 & $1 /(\mathrm{n}-1)$ & $1 /(\mathrm{n}-1)$ & $\ldots$ & 1 & 1 \\
\hline$\ldots$ & $\ldots$ & $\ldots$ & $\ldots$ & $\ldots$ & $\ldots$ & $\ldots$ \\
\hline$\ldots$ & $\ldots$ & $\ldots$ & $\ldots$ & $\ldots$ & $\ldots$ & $\ldots$ \\
\hline$(\mathrm{n}-2) /(\mathrm{n}-1)$ & 0 & $1 /(\mathrm{n}-1)$ & $2 /(\mathrm{n}-1)$ & $\ldots$ & 1 & 1 \\
\hline 1 & 0 & $1 /(\mathrm{n}-1)$ & $2 /(\mathrm{n}-1)$ & $\ldots$ & $(\mathrm{n}-2) /(\mathrm{n}-1)$ & 1 \\
\hline
\end{tabular}

It is obvious that Rosser truth values also determine a contact algebra.

Remark 3.2. (a) Every natural number $n$ determines a contact algebra. If $n$ is a prime, the corresponding di-contact algebra is Boolean.(whitesitt,1962)

(b) A natural number $n=\{0,1,2,3, \ldots, n-1\}$ is a chain contact algebra.

Remark 3.3. Lukasiewicz contact algebra is much more attractive both for physicists as well as mathematicians since contacts are in continuously increasing order of magnitudes from 0 to 1 .

\section{Fuzzy model of contact algebra}

In our model the concept of contact values is a generalization of the notion of contact values in Boolean circuits. In fact, the number of occurrence of contact values of a contact and their order of occurrence is significant in a circuit. Whether by design or by electrical expediency, the technicians have done a lot so that the involvement of different types of contact with different contact values in an electric circuit has become inevitable (Tripathi, 2004). We observe even today that a contact is not always two valued. For instance, a regulator may contain any number of contact values. As such, in an electric circuit the contact values 0 , $1,2,3, \ldots$, which may be finite or infinite, arranged in the usual order of each of contact are kept in a set $L$ which we have called a list. The symbol $P_{L}^{(x)}$ will represent the list of values of a contact $x$ with respect to its position and $X$, the set of all contacts involved in a circuit (Pathak et al, 2017).

Here we have defined two basic operations 'on' and 'off' on a list $L$ and established some results involving them. The respective versions of these operations on a fuzzy list have been introduced and the properties are evaluated (Zhang et al, 2009).

Definition 4.1. Let $L$ be a list drawn from $X$. Then we define on-operator (in short O-operator) on $L$ such that for every $n \in N$, $O(n, L)$ is defined by the position function :

$$
P_{O(n, L)}^{(x)}=\phi \text { or }\left\{r: r<n \text { and } r \in P_{L}^{(x)}\right\}
$$

According as $n \leq \min P_{L}^{(x)}$ or $P_{L}^{(x)}=\phi$ or $n \leq \min P_{L}^{(x)}$ for any $x \in L$. 
Here $P_{L}^{(x)}=\phi$ essentially means that $x \in X$ acts as an insulator in a circuit.

Definition 4.2. Let $L$ be a list drawn from $X$. Then we define off-operator (in short f-operator) on $L$ such that for every $n \in N, f(n, L)$ is defined by the position function :

$$
P_{f(n, L)}^{(x)}=\phi \text { or }\left\{r-n: r \geq n \text { and } r \in P_{L}^{(x)}\right\}
$$

According as $n \leq \max P_{L}^{(x)}$ or $P_{L}^{(x)}=\phi$ or $n \leq \min P_{L}^{(x)}$ for any $x \in X$.

Definition 4.3. A set $L^{\prime}$ is said to be an initial sublist of a list $L$ iff for some $n \in N$,

$$
L^{\prime}=O(n, L) .
$$

Definition 4.4. A set $L^{\prime}$ is said to a terminal sublist of $L$ iff for some $n \in L$,

$$
L^{\prime}=f(O, L) \text {. }
$$

Theorem 4.1. For any list $L$ drawn from $X$ and $n \in N$,

$$
O(n, L) H f(n, L)=L,
$$

Where $O(n, L)+f(n, L)$ stands for the position function

$$
P_{O(n, L) H f(n, L)}^{(x)}=P_{O(n, L)}^{(x)} \cup\left\{\mid O\left(n, L \mid+t: t \in P_{f(n, L)}^{(x)}\right\} .\right.
$$

Proof. Let $x \in X$. Then three cases occur.

Case 1. For $n \leq \max P_{L}^{(x)}$ : In this case

$$
\begin{aligned}
P_{O(n, L) H f(n, L)}^{(x)} & =\phi \bigcup\left\{n+t: t \in P_{f(n, L)}^{(x)}\right\} \\
& =\left\{n+t: t \in\left\{r-n: r \geq n \text { and } r \in P_{L}^{(x)}\right\}\right\} \\
& \left.=\left\{n+(r-n): r \in P_{L}^{(x)}\right\}\right\} \quad\left(n \leq \min P_{L}^{(x)} \text { and } r \in P_{L}^{(x)}\right) \\
& \left.=r: r \in P_{L}^{(x)}\right\} \\
& \left.=P_{L}^{(x)}\right\}
\end{aligned}
$$

Case 2. For $n>\max P_{L}^{(x)}$ : Here

$$
\begin{aligned}
P_{O(n, L) H f(n, L)}^{(x)}= & \left\{r: r<n \text { and } r \in P_{L}^{(x)}\right\} \\
& \left(\left\{\min (n,|L|)+t: t \in P_{L}^{(x)}\right\}=\phi \text { and since } n>r, \forall r \in P_{L}^{(x)}\right) \\
= & \left.r: r \in P_{L}^{(x)}\right\} \\
P_{O(n, L) H f(n, L)}^{(x)} & \left.\left\{P_{L}^{(x)}:\right\} \in P_{L}^{(x)} \text { and } r<n\right\} \\
& \left(\left\{\min (n,|L|)+t: t \in P_{L}^{(x)}\right\}=\phi \text { and since } n>r, \forall r \in P_{L}^{(x)}\right)
\end{aligned}
$$

Case 3. For $\min P^{(x)}<n \leq \max P_{L}^{(x)}$ : Here

$$
\begin{aligned}
& \bigcup\left\{n+t: t \in\left\{r-n: r \geq n \text { and } r \in P_{L}^{(x)}\right\}\right\} \\
& \left(|L| \geq \max P_{L}^{(x)} \Rightarrow \min (n,|L|=n)\right.
\end{aligned}
$$


Remark 4.1. For any finite list $L$ and $m, n \in N, f(m, f(n, L))=f(m+n, L)$.

Remark 4.2. For any list $L$ drawn from $X, t 1(L)=f(1, L)$.

Remark 4.3. For any list $L$ drawn from $X$, init $(L)=O(|L|-1, L)$.

Remark 4.4. For any list $L$ drawn from $X$ and $n \in N$,

$$
f(n, L)=L-O(n, L) \text {. }
$$

Remark 4.5. For any list $L$ drawn from $X$ and $n \in N$,

$$
O(n, L)=L-f(n, L)
$$

Remark 4.6. For any list $L$ drawn from $X$ and $m, n \in N$,

$$
O(m, f(n, L))=O(m+n, L)-O(n, L) .
$$

Remark 4.7. For any list $L$ drawn from $X$ and $m, n \in N$,

$$
f(m, O(n, L))=O(n, L)-O(m, L) \text {. }
$$

Corollary 4.1. For any list $L$ drawn from $X$ and non-negative integers $m_{1}, m_{2}, m_{3}, \ldots, m_{k}$, we have

$O\left(m_{1}, O\left(m_{2}, \ldots, O\left(m_{k}, L\right)\right)\right)=O(\rho, L)$,

where $\rho=\min \left(m_{1}, m_{2}, m_{3}, \ldots, m_{k}\right)$.

Corollary 4.2. For any list $L$ drawn from $X$, and $m, n \in N$,

$$
O(m, f(n, L))=f(n, O(m+n, L)) .
$$

Theorem 4.2. For any list $L$ and $m, n \in N$

$$
O(m, O(n, L))=O(\rho, L)
$$

where $\rho=\min (m, n)$.

Proof. Here three cases arise.

Case 1. $m>n$. Here $\rho=n$. So, it is required to establish that

$$
O(m, O(n, L))=O(n, L) \text {. Let } L^{\prime}=O(n, L)
$$

$m>\left|L^{\prime}\right|$, since $|O(n, L)| \in n$. Now, for any $x \in X$, by definition of 'on'

$$
P_{O\left(m, L^{\prime}\right)}^{(x)}=P_{L^{\prime}}^{(x)}=P_{O(n, L)}^{(x)}=P_{L}^{(x)} \text { So, } P_{O(m, O(n, L))}^{(x)}=P_{O(n, L)}^{(x)} \forall x \in X
$$

Case 2. $m=n$. Here we have to show that

$$
O(n, O(n, L))=O(n, L)
$$

If $n<|L|$, then $O(n, L)=L$. So, $O(n, O(n, L))=O(n, L)$. If $n<|L|$, then two subcases arise.

Subcase (a) $n \leq \min P_{L}^{(x)}$. Here $P_{O(n, L)}^{(x)}=\phi$. So, $P_{O(n, O(n, L))}^{(x)}=\phi=P_{O(n, L)}^{(x)}$

Subcase (b) $n \leq \min P_{L}^{(x)}$. Here $P_{L}^{(x)}=\left\{r: r<n\right.$ and $\left.r \in P_{L}^{(x)}\right\}$

Now, $\quad P_{O(n, O(n, L))}^{(x)}=r: r<n$ and $\left.r \in P_{L}^{(x)}\right\}$ 


$$
\begin{aligned}
& =\left\{r: r<n \text { and } r \in\left\{t: t<n \text { and } t \in P_{L}^{(x)}\right\}\right\} \\
& =\left\{t: t<n \text { and } t \in P_{L}^{(x)}\right\}
\end{aligned}
$$

This proves that $O(n, O(n, L))=O(n, L)$ in this case.

Case 3. $m<n$. Here we have to show that $\mathrm{O}(m, O(n, L))=O(m, L)$.

(i) Suppose, $|L|<m$. Then $|L|<m<n$. So,

$$
P_{O(n, L)}^{(x)}=P_{L}^{(x)} \text { and } P_{O(m, L)}^{(x)}=P_{L}^{(x)}
$$

So, $P_{O(m, O(n, L))}^{(x)}=P_{O(m, L)}^{(x)}$

$$
\begin{aligned}
& =P_{L}^{(x)} \\
& =P_{O(n, L)}^{(x)}=P_{O(m, L)}^{(x)}
\end{aligned}
$$

Hence, $O(m, O(n, L))=O(m, L)$.

(ii) $m<|L|<n$. We have to show that

$$
P_{O(m, O(n, L))}^{(x)}=P_{O(m, L)}^{(x)}
$$

Since $n>|L|, O(n, L)=L$.

Hence the proof follows.

(iii) $m<n<|L|$. We have to show that

$$
O(m, O(n, L))=O(m, L)
$$

If we take $O(n, L))=\bigcup$, the proof is direct as both the sides are $\bigcup$. Otherwise two subcases arise.

Subcase (a) In this subcase $P_{O(m, L)}^{(x)}=\phi$. Also

$$
P_{O(m, O(n, L))}^{(x)}=\phi, \text { since } P_{O(n, L)}^{(x)} \subseteq P_{L}^{(x)}
$$

and hence $m \leq \min P_{O(n, L)}^{(x)}$. So,

$$
P_{O(m, O(n, L))}^{(x)}=P_{O(m, L)}^{(x)}, \forall x \in \phi .
$$

Subcase (b) $\min P_{O(n, L)}^{(x)}<m<n|L|$. We have to show that

$$
P_{O(m, O(n, L))}^{(x)}=P_{O(m, L)}^{(x)}
$$

We have 


$$
\begin{aligned}
P_{O(m, O(n, L))}^{(x)} & =\left\{t: t<m \text { and } t \in P_{O(n, L)}^{(x)}\right\} \\
& =\left\{t: t<m \text { and } t \in P_{L}^{(x)}\right\} \\
& =P_{O(m, L)}^{(x)}
\end{aligned}
$$

This completes the proof.

Corollary 4.3. For any list $L$ drawn from $X$ and non-negative integers $m_{1}, m_{2}, m_{3}, \ldots, m_{k}$ we have

$O\left(m_{1}, O\left(m_{2}, \ldots,\left(O\left(m_{k}, L\right)\right)=O(\rho), L\right)\right.$,

where $\rho=\min \left(m_{1}, m_{2}, m_{3}, \ldots, m_{k}\right)$.

Remark 4.8. For any list $L$ drawn from $X$ and $n \in N$,

$$
f(n, L)=L-O(n, L) .
$$

Remark 4.9. For any list $L$ drawn from $X$ and $n \in N$,

$$
O(n, L)=L-f(n, L)
$$

Remark 4.10. For any list $L$ drawn from $X$ and $m, n \in N$,

$$
O(m, f(n, L))=O(m+n, L)-O(n, L) .
$$

Remark 4.11. For any list $L$ drawn from $X$ and $m, n \in N$,

$$
f(m, O(n, L))=O(n, L)-O(m, L) .
$$

Corollary 4.4. For any list $L$ drawn from $X$ and $m, n \in N$,

$$
O(m, f(n, L))=f(n, O(m+n, L)) .
$$

\section{Conclusions}

All the results on lists with two operations 'on' and 'off' can be extended to the setting of fuzzy lists. The properties of 'on' and 'off' operators provide a complete structure on the application to switches in a circuit. So, some new properties of 'on' and 'off' operators can be established. The parallel concept of these two operators on fuzzy lists can also be introduced. The study can be carried out further by introducing certain results on lists and fuzzy lists.

\section{Acknowledgments}

We are very grateful to the Department of Mathematics, Amrit Campus and Central Department of Mathematics, Tribhuvan University, Kathmandu, Nepal, for their continuous support for research activities and encouragement.

\section{References}

Birkhoff, G. 1984. Lattice Theory, AMS coll. Publication, vol. 25, Providence, Rhode Island.

Bolyar, J. 1981. Finite Algebra and multiple valued logic. North Halland, Amsterdam, 825-872.

Lafont,Y. 2003. Towards an algebraic theory of Boolean Circuits. J. of Pure and Applied Algebra. 11 (01).

Lafont,Y. 2004. Towards an algebraic theory of Boolean Circuits. J. of Pure and Applied Algebra, 184 (23), 257-310. 
Lal, R.N. and Jha, J.S. 1998. Browerian Switching Algebra, J. of the Bhagalpur University natural science, vol. VIII, No 1, 7575.

Meckinsey, J.C.C. and Tarski, A. 1947. On closed elements in closure Algebra, Ann of math vol. 46, AMS, 122-162.

Pathak, S.R. and Yadav, R.N. 2017. Graph theory and networks. Eureka, 4 (1- 4), 2392- 4233.

Steiner, A.K. 1966. The lattice of topologies: structure and complementation. Trans. Amer. Math. Soc., 122(2):379-398.

Tripathi, B., Pattanaik, G.P. 2004. On some properties of lists and fuzzy lists. Journal of Information sciences, vol, 168, issue, 1-4 Wallman, H. 1938. Lattices and topological spaces. Math. Ann. 39:112-136.

Whitesitt, J.E. 1962. Boolean Algebra and its Applications. Addison-Wesley Pub.Com. INC.

Zhang, Q., Xie, W. and Fan, L. 2009. Fuzzy Sets and System; Aveuue of America, 160 (16) 